\title{
Synthesis of $\mathrm{CoFe}_{2} \mathrm{O}_{4}$ Nanoparticles: The Effect of Ionic Strength, Concentration, and Precursor Type on Morphology and Magnetic Properties
}

\author{
Izabela Malinowska, ${ }^{1}$ Zuzanna Ryżyńska, ${ }^{2}$ Eryka Mrotek, ${ }^{1}$ Tomasz Klimczuk, ${ }^{2}$ \\ and Anna Zielińska-Jurek (iD) \\ ${ }^{1}$ Department of Process Engineering and Chemical Technology, Gdansk University of Technology, 80-233 Gdansk, Poland \\ ${ }^{2}$ Department of Solid State Physics, Faculty of Applied Physics and Mathematics, Gdansk University of Technology, \\ 80-233 Gdansk, Poland
}

Correspondence should be addressed to Anna Zielińska-Jurek; annjurek@pg.edu.pl

Received 9 December 2019; Accepted 7 April 2020; Published 5 May 2020

Academic Editor: Leander Tapfer

Copyright (c) 2020 Izabela Malinowska et al. This is an open access article distributed under the Creative Commons Attribution License, which permits unrestricted use, distribution, and reproduction in any medium, provided the original work is properly cited.

\begin{abstract}
The present study highlights the effect of metal precursor types $\left(\mathrm{SO}_{4}{ }^{2-}, \mathrm{Cl}^{-}\right.$, and $\left.\mathrm{NO}_{3}{ }^{-}\right)$, their concentration, and the influence of ionic strength of reaction environment on the morphology, surface, and magnetic properties of $\mathrm{CoFe}_{2} \mathrm{O}_{4}$ particles. The magnetic nanoparticles were obtained by chemical coprecipitation in alkaline medium at increasing metal concentration in the range of $0.0425 \mathrm{~mol} \cdot \mathrm{dm}^{-3}$ to $0.17 \mathrm{~mol} \cdot \mathrm{dm}^{-3}$ and calcination temperature from $400^{\circ} \mathrm{C}$ to $800^{\circ} \mathrm{C}$. It was found that the chemistry of precursors can be directly correlated with magnetic properties. The $\mathrm{CoFe}_{2} \mathrm{O}_{4}$ particles from metal sulphate precursors showed the highest saturation magnetization and the lowest coercivity. The adjustment of ionic strength in the range of $1.25-5 \mathrm{M}$ was achieved by adding an appropriate quantity of metal sulphates into aqueous solutions at a constant $\mathrm{pH}$ or by adding an appropriate quantity of $\mathrm{NaClO}_{5}$ under similar conditions. The average hydrodynamic size of $\mathrm{CoFe}_{2} \mathrm{O}_{4}$ increased from $46 \mathrm{~nm}$ to $54 \mathrm{~nm}$ with increasing metal concentration and ionic strength. An explanation of magnetic properties, caused by ionic strength and metal concentration, is given based mainly on the reduction in repulsive forces at the particle interface and compensation of the double electric layer in the presence of anions. The observed coercivity was lower for the particles obtained in solutions with the highest ionic strength, whereas the concentration of metals and calcination temperature affected the saturation magnetization and morphology of the obtained cobalt ferrite particles.
\end{abstract}

\section{Introduction}

Spinel ferrites are interesting dielectric materials due to their magnetic properties and high chemical and thermal stability. In recent years cobalt ferrite nanomaterials have gained considerable attention due to their unique electric, catalytic, magnetic, optical, and medical properties $[1,2]$, which determine their potential applications in catalysis, gas sensors, information storage systems, medical diagnostics, and therapy [3-7]. Currently, research is also focused on the use of cobalt ferrites for the separation of magnetic photocatalysts after water treatment processes [8] and separation of bacteria [7]. The advantage of $\mathrm{CoFe}_{2} \mathrm{O}_{4}$ nanoparticles is one order larger of crystalline anisotropy compared to magnetite with the same saturation magnetization. Therefore, the magnetic moment of cobalt ferrite relaxes much slower than of magnetite or maghemite with similar particle size $[9,10]$.

Nanoparticles of $\mathrm{CoFe}_{2} \mathrm{O}_{4}$ were prepared by a great number of methods: sol-gel $[11,12]$, hydrothermal [13], microemulsion [14], aqueous precipitation [15], polyol [16], and combustion [17]. The characterization of the physicochemical properties was mainly focused on the correlation between particle size and magnetic properties. However, no systematic study on the effect of cobalt and iron ion precursor types, the concentration of metal ions in the reaction environment, and the ionic strength on the magnetic properties of cobalt 
ferrite nanoparticles has been reported. The understanding of such relationships is an important requirement to attain the capability of tailoring the properties of the cobalt ferrite-based magnetic materials.

In this regard, the present study focuses on the magnetic properties of cobalt ferrite obtained in the hydrothermal process at calcination temperatures in the range of $400-800^{\circ} \mathrm{C}$ using varying salt type precursors. For the first time, the effect of metal precursors (sulphates, chlorides, and nitrates) and their concentration used for the synthesis of cobalt ferrite NPs, the influence of ionic strength of reaction environment, and the calcination temperature on size, structural, and magnetic properties of $\mathrm{CoFe}_{2} \mathrm{O}_{4}$ particles were studied.

\section{Experimental}

2.1. Preparation of $\mathrm{CoFe}_{2} \mathrm{O}_{4}$ Particles. All the reagents used in the experiments were of analytical grade and used without further purification. Cobalt(II) nitrate hexahydrate, iron(III) nitrate nonahydrate, iron(III) chloride hexahydrate, cobal$\mathrm{t}$ (II) chloride hexahydrate, iron(III) sulphate heptahydrate, cobalt(II) sulphate heptahydrate, and sodium hydroxide were purchased from Aldrich, Germany. Cobalt ferrite particles were obtained using a precipitation method combined with hydrothermal reaction at a constant $\mathrm{pH}$ of 12 . In this regard, $20 \mathrm{~cm}^{3}$ of cobalt salt solution and $40 \mathrm{~cm}^{3}$ of iron salt solution were mixed in a stoichiometric 2:1 (Fe: Co) molar ratio under stirring $(250 \mathrm{rpm})$ for 30 minutes. Then, $110 \mathrm{~cm}^{3}$ of $5 \mathrm{M} \mathrm{NaOH}$ was prepared and dropwise added at room temperature into obtained brown metal salt solution during mixing, while the $\mathrm{pH}$ was constantly monitored. The reactants were stirred for 30 minutes using a magnetic stirrer $(250 \mathrm{rpm})$ until the $\mathrm{pH}$ was 12 . Then, the resulting colloid was transferred into a $200 \mathrm{~cm}^{3}$ Teflon lined stainless steel autoclave. The hydrothermal reaction was performed at $200^{\circ} \mathrm{C}$ for $5 \mathrm{~h}$, and the obtained suspension was washed with water several times and separated by magnetic decantation. Finally, the obtained $\mathrm{CoFe}_{2} \mathrm{O}_{4}$ particles were dried at $120^{\circ} \mathrm{C}$ to dry mass and calcined at $400-800^{\circ} \mathrm{C}$ for $2 \mathrm{~h}$. The adjustment of ionic strength in the range of $1.25-5 \mathrm{M}$ was achieved by adding the appropriate quantity of metal sulphates into aqueous solutions with a constant $\mathrm{Fe}$ : Co molar ratio of $2: 1$ or by adding the appropriate quantity of $\mathrm{NaClO}_{5}$ under similar conditions.

2.2. Characterization of $\mathrm{CoFe}_{2} \mathrm{O}_{4}$ Particles. Magnetic measurements (magnetization, remanence, and coercivity) were performed using Physical Property Measurement System (PPMS) (Quantum Design, San Diego, CA, USA) at the temperature of $293 \mathrm{~K}$ and in the range of $0-3 \mathrm{~T}$.

XRD analysis was performed to characterize the crystallinity of the as-obtained cobalt ferrite nanoparticles. In this regard, a Rigaku MiniFlex 600 X-ray diffraction system equipped with a sealed tube $\mathrm{X}$-ray generator was used. Data acquisition conditions were as follows: $2 \theta$ range $5-80^{\circ}$, scan speed $1^{\circ} \cdot \mathrm{min}^{-1}$, and scan step $0.01^{\circ}$. The crystallite size of the ferrite particles in the direction vertical to the corresponding lattice plane was determined using Scherrer's equation based on the corrected full width at half maximum
(FWHM) of the XRD peak and angle of diffraction. The subtraction of the FWHM of the standard was employed as a correction method. The crystalline and amorphous phase content was analyzed using an internal standard $(\mathrm{NiO}$, Aldrich, Germany).

To evaluate the light absorption properties of the obtained $\mathrm{CoFe}_{2} \mathrm{O}_{4}$ particles, the diffuse reflectance (DR) spectra were recorded using a Jasco V-670 spectrophotometer equipped with a PIN-757 integrating sphere. $\mathrm{BaSO}_{4}$ was used as a reference. The band gap energies were calculated from the corresponding Kubelka-Munk function, $F(R)$, which is proportional to the absorption of radiation, by plotting $F(R)^{0.5} E_{\mathrm{ph}} 0.5$ against $E_{\mathrm{ph}}$, where $E_{\mathrm{ph}}$ is the photon energy.

Nitrogen adsorption-desorption isotherms were recorded at liquid nitrogen temperature $(77 \mathrm{~K})$ using the Micromeritics Gemini V (model 2365) instrument, and the specific surface areas were determined using the Brunauer-Emmett-Teller (BET) method. Surface charges (zeta potential) and particles size were measured using a Nano-ZS Zetasizer dynamic light scattering detector (Malvern Instruments, UK) equipped with a $4.0 \mathrm{~mW}$ internal laser. Infrared (IR) reflection spectra of cobalt ferrites were measured within a $500-5000 \mathrm{~cm}^{-1}$ range by employing a Fourier transform infrared (FTIR) spectrometer Nicolet 8700 (Thermo) equipped with a single reflection diamond crystal.

\section{Results and Discussion}

3.1. The Effect of Metal Precursor Types. While the effect of surfactants and reaction conditions has been widely investigated, there is no information on the effect of metal precursor types and their concentration on the formation of $\mathrm{CoFe}_{2} \mathrm{O}_{4}$ nanoparticles. Therefore, in this study, the effect of metal precursors (sulphates, chlorides, and nitrates) used for the synthesis of cobalt ferrite NPs was investigated.

The BET surface area for samples S1, S2, and S3 varied from $31 \mathrm{~m}^{2} \cdot \mathrm{g}^{-1}$ to $50 \mathrm{~m}^{2} \cdot \mathrm{g}^{-1}$. The sample from metal nitrate precursors (S2) revealed the smallest surface area, while sample S3 from chloride metal precursors showed the highest BET surface area (see Table 1). The calculated BET equivalent primary particle diameter for the samples S1, S2, and S3, which differ in precursor types (S1-sulphates, S2-nitrates, and S3-chlorides) was $25 \mathrm{~nm}, 37 \mathrm{~nm}$, and $23 \mathrm{~nm}$, respectively. The phase and structural analyses were performed by employing X-ray diffraction (XRD) measurements and are presented in Figure 1.

The presence of (220), (311), (400), (422), (511), and (440) in XRD patterns is in accordance with inverse cubic spinel structure with space group fd-3m, which is in agreement with JCPDS standard card no. 01-077-0426. In $\mathrm{CoFe}_{2} \mathrm{O}_{4}$, the divalent cation of $\mathrm{Co}^{2+}$ occupied the octahedral position, while the $\mathrm{Fe}^{3+}$ ions are located at the tetrahedral and octahedral sites. The lattice parameter obtained from Rietveld analysis was found to be $8.39 \AA$. The samples S1-S3 obtained without heat treatment contained $80 \%$ (S1), 90\% (S2), and $85 \%$ (S3) amorphous phase content; hence, the highest crystallinity of $20 \%$ was observed for cobalt ferrite obtained from sulphates (sample S1). Previously, Zhao et al. 
TABLE 1: The effect of precursor types on physicochemical and magnetic properties of $\mathrm{CoFe}_{2} \mathrm{O}_{4}$.

\begin{tabular}{|c|c|c|c|c|c|c|c|c|c|c|}
\hline $\begin{array}{l}\text { Sample } \\
\text { labeling }\end{array}$ & Precursors & $M_{\mathrm{s}}\left(\mathrm{emu} \cdot \mathrm{g}^{-1}\right)$ & $H_{\mathrm{c}}(\mathrm{T})$ & $M_{\mathrm{r}}\left(\mathrm{emu} \cdot \mathrm{g}^{-1}\right)$ & $A_{\mathrm{BET}}\left(\mathrm{m}^{2} \cdot \mathrm{g}^{-1}\right)$ & $V_{\mathrm{p}}\left(\mathrm{cm}^{3} / \mathrm{g}\right)$ & $D_{\mathrm{XRD}}(\mathrm{nm})$ & $D_{\mathrm{BET}}(\mathrm{nm})$ & $D_{\text {DLS }}(\mathrm{nm})$ & $E_{\mathrm{g}}$ \\
\hline S1 & $\begin{array}{c}\text { Suplhates } \\
\mathrm{CoSO}_{4} \cdot 7 \mathrm{H}_{2} \mathrm{O} \\
\mathrm{Fe}_{2}\left(\mathrm{SO}_{4}\right)_{3} \cdot 7 \mathrm{H}_{2} \mathrm{O}\end{array}$ & 60 & 0.007 & 9.0 & 45 & 0.024 & 15 & 25 & 40 & 1.80 \\
\hline S2 & $\begin{array}{c}\text { Nitrates } \\
\mathrm{Co}\left(\mathrm{NO}_{3}\right)_{2} \cdot 6 \mathrm{H}_{2} \mathrm{O} \\
\mathrm{Fe}\left(\mathrm{NO}_{3}\right)_{3} \cdot 9 \mathrm{H}_{2} \mathrm{O}\end{array}$ & 50 & 0.027 & 11.6 & 31 & 0.015 & 20 & 37 & 47 & 1.72 \\
\hline S3 & $\begin{array}{l}\text { Chlorides } \\
\mathrm{CoCl}_{2} \cdot 6 \mathrm{H}_{2} \mathrm{O} \\
\mathrm{FeCl}_{3} \cdot 6 \mathrm{H}_{2} \mathrm{O}\end{array}$ & 50 & 0.028 & 9.8 & 50 & 0.025 & 14 & 23 & 37 & 1.80 \\
\hline
\end{tabular}

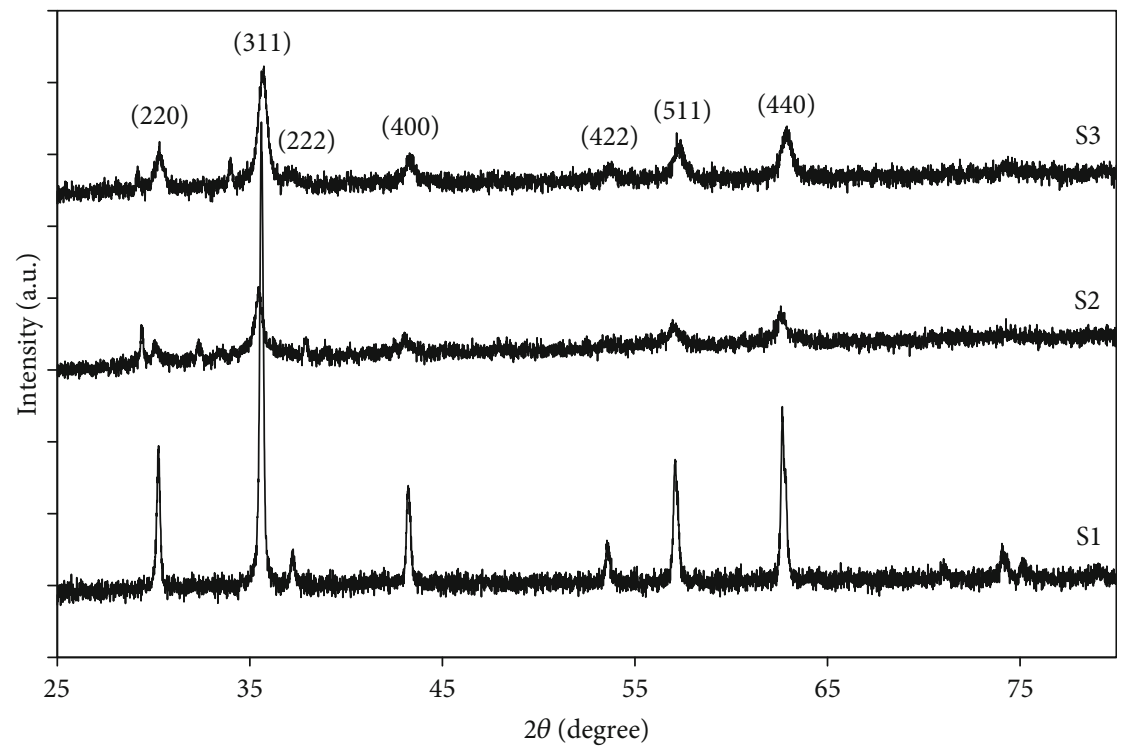

FIGURE 1: X-ray diffraction patterns of the as-prepared cobalt ferrite particles. The effect of precursor types: S1-sulphates, S2-nitrates, and S3-chlorides.

found that low-crystalline mesoporous cobalt ferrite particles possess improved electrochemical properties [18]. The average size of crystallites calculated from XRD by employing the Debye-Scherrer formula for S1, S2, and S3 samples was about $15 \mathrm{~nm}, 20 \mathrm{~nm}$, and $14 \mathrm{~nm}$, respectively. Based on dynamic light scattering (DLS) analysis, hydrodynamic particle size was determined. It was found that larger particles were produced using nitrates as metal precursors than from sulphates and chlorides, as shown in Table 1.

The observed difference in particle size between samples S1, S2, and S3 may derive from the structural properties and lattice strain as a result of the clustering of the nanoparticles. The particle sizes determined from DLS analysis are larger than calculated based on XRD measurements and the equivalent diameter calculated from the BET surface area. It can be explained that based on DLS analyses, hydrodynamic size of particles and their surrounding diffuse layer is determined, whereas XRD calculation gives the crystallite size of $\mathrm{CoFe}_{2} \mathrm{O}_{4}$ [19]. However, the particle may contain one or more grains. Therefore, the particle size is expected in between crystallite size and hydrodynamic diameter. That was confirmed by SEM microscopy analysis (see Figure 2). The average particle size calculated from the statistical average size distribution of $100 \mathrm{CoFe}_{2} \mathrm{O}_{4} \mathrm{NPs}$ was about $32 \mathrm{~nm}, 37 \mathrm{~nm}$, and $29 \mathrm{~nm}$ for S1, S2, and S3, respectively. The particle size from the microscopy analyses corresponded well with the BET equivalent diameter calculated according to the $d=6 /\left(A_{\mathrm{BET}} \bullet \rho\right)$ equation.

The zeta potential values of cobalt ferrite suspensions are shown in Figure 3. As presented, their magnitudes strongly depended on the $\mathrm{pH}$ of the aqueous phase. At low $\mathrm{pH}$ values, $\mathrm{H}^{+}$ions are adsorbed on the particle surface, and therefore, it is positively charged, while at $\mathrm{pH}$ values above the isoelectric point (IEP), a surface is negatively charged as a consequence of $\mathrm{OH}^{-}$ion adsorption on the particle surface. Under acidic conditions, the highest electrostatic stability was observed for sample S3 with a zeta potential value of $+42.6 \mathrm{mV}$ at $\mathrm{pH}=4$. On the other hand, in alkaline conditions, the highest value of zeta potential was $-50.9 \mathrm{mV},-48.0 \mathrm{mV}$, and $-47.0 \mathrm{mV}$ observed at $\mathrm{pH}=12$ for S1, S2, and S3, respectively. That means all obtained cobalt ferrite particles are stable at $\mathrm{pH}$ above 9, and only 


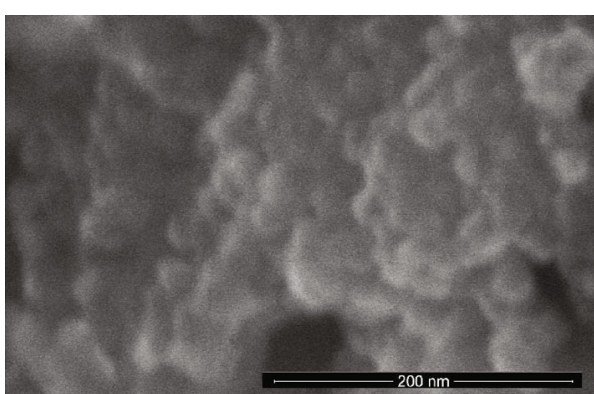

(a)

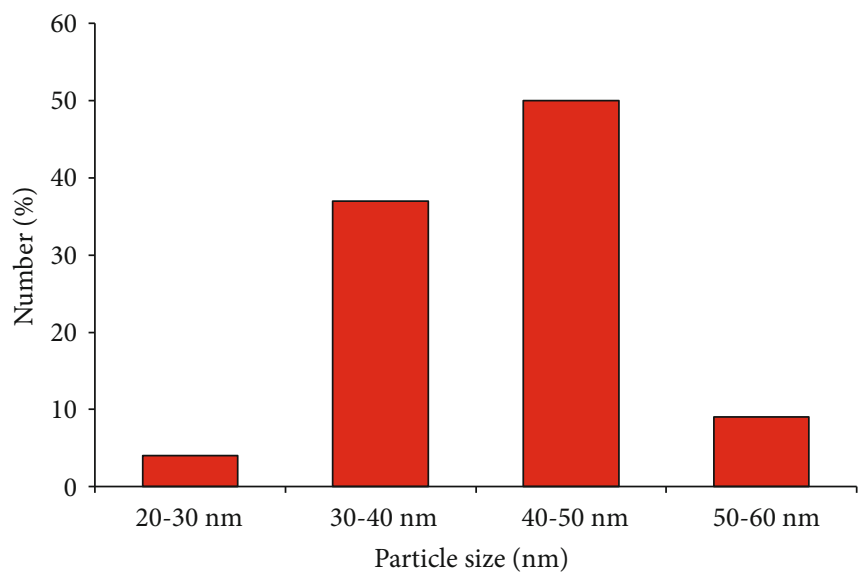

(c)

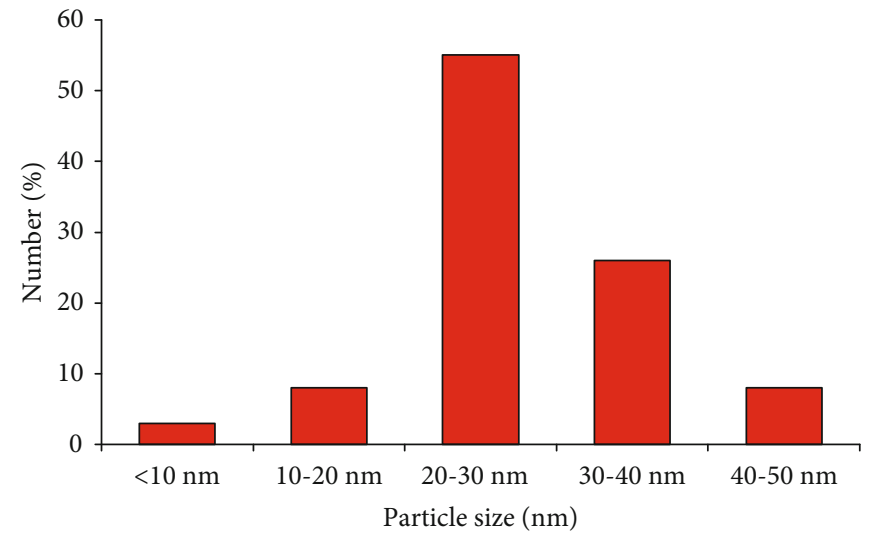

(b)

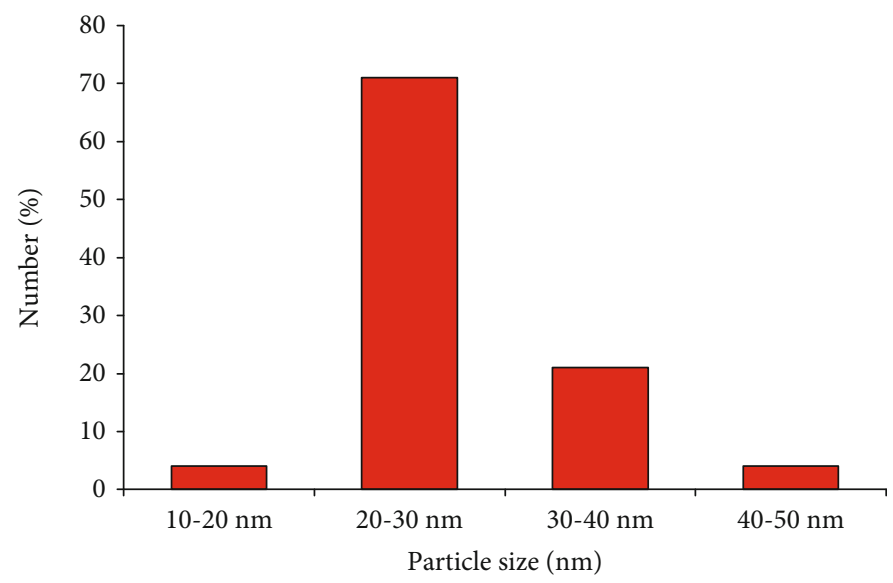

(d)

FIGURE 2: SEM image of $\mathrm{CoFe}_{2} \mathrm{O}_{4}$ particles (a) and size distribution for samples S1, S2, and S3 synthesized using metal precursors: sulphates (b), nitrates (c), and chlorides (d).

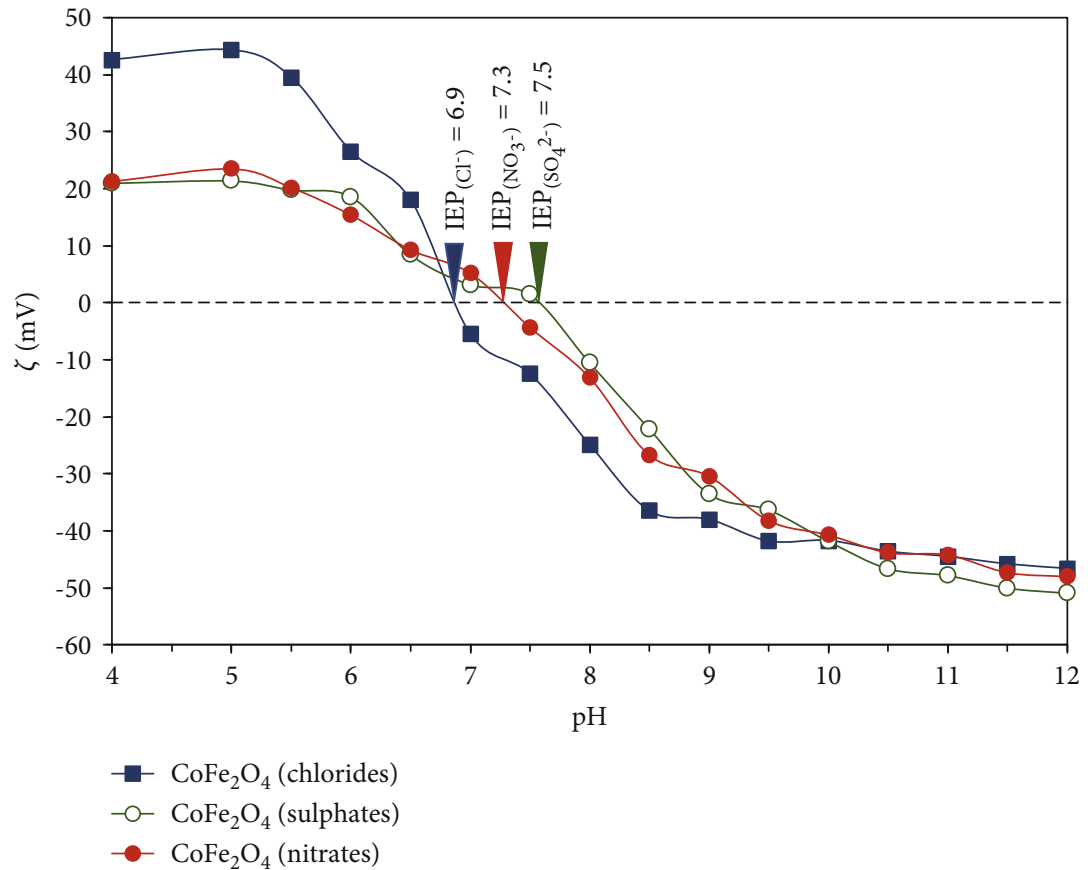

FIGURE 3: Electrophoretic mobility of $\mathrm{CoFe}_{2} \mathrm{O}_{4}$ suspensions $\left(0.1 \mathrm{~g} \mathrm{dm}^{-3}\right)$ vs. $\mathrm{pH}\left(I=1 \cdot 10^{-2}\right)$. 


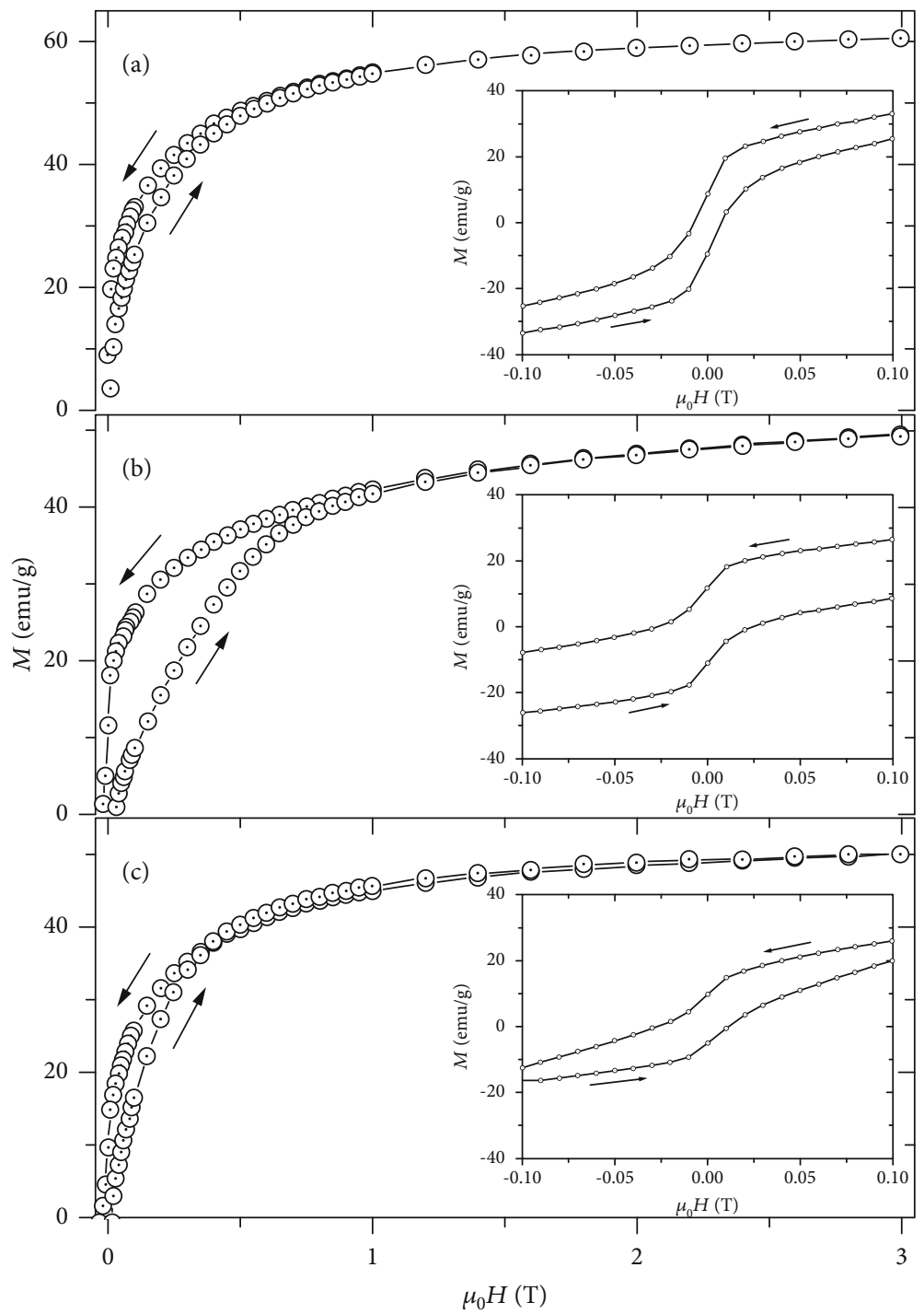

Figure 4: Hysteresis loops of nanoparticles of $\mathrm{CoFe}_{2} \mathrm{O}_{4}$ synthesized using metal precursors: (a) sulphates, (b) nitrates, and (c) chlorides.

the one obtained from chlorides can be stable under acidic conditions $(\mathrm{pH}<6.5)$. However, in order to enhance the separation capability of colloidal particles from the aqueous phase, the process should proceed near the isoelectric point (IEP). Minor differences in values of the isoelectric point were observed among the samples. For $\mathrm{CoFe}_{2} \mathrm{O}_{4}$ from sulphates, IEP occurred at $\mathrm{pH}=7.5$, while for $\mathrm{CoFe}_{2} \mathrm{O}_{4}$ obtained from chloride precursors, it was found at $\mathrm{pH}=6.9$.

The Tauc transformation of DR/UV-Vis spectra allows determining the optical band gap energies of $\mathrm{CoFe}_{2} \mathrm{O}_{4}$ nanoparticles. The particles from metal nitrate precursors exhibited lower $E_{\mathrm{g}}=1.72 \mathrm{eV}$ than cobalt ferrite particles $\left(E_{\mathrm{g}}=1.8 \mathrm{eV}\right)$ synthesized from sulphate or chloride metal precursors (see Table 1).

All three samples exhibited room temperature ferrimagnetism with different saturation, remanent, and coercive values. The hysteresis loops obtained from VSM measurements for $\mathrm{CoFe}_{2} \mathrm{O}_{4}$ particles are presented in Figure 4. All the hysteresis loops are typical for soft magnetic materials, and an "S" shape of the curves together with low coercivity $\left(H_{\mathrm{c}}=0.007 \mathrm{~T}\right.$ for S1 and $0.028 \mathrm{~T}$ for S2 and S3) indicates the presence of small magnetic particles. The saturation magnetization $\left(M_{\mathrm{s}}\right)$ and coercivity $\left(H_{\mathrm{c}}\right)$ values of the ferrite particles are listed in Table 1.

The chemistry of precursors was directly correlated with magnetic properties. The $\mathrm{CoFe}_{2} \mathrm{O}_{4}$ particles from metal sulphate precursors showed the highest saturation magnetization $\left(M_{\mathrm{s}}\right)$ of $60 \mathrm{emu} \cdot \mathrm{g}^{-1}$, the lowest remanent magnetization $\left(M_{r}\right)$ of $9 \mathrm{emu} \cdot \mathrm{g}^{-1}$, and the coercivity $\left(H_{\mathrm{c}}\right)$ at $0.007 \mathrm{~T}$. In view of this, the metal sulphate precursors were used for further studies of the effect of preparation parameters, e.g., concentration of metals, ionic strength of a solution, and calcination temperature on physicochemical properties of $\mathrm{CoFe}_{2} \mathrm{O}_{4}$ particles.

3.2. The Effect of Concentration, Ionic Strength, and Calcination Temperature. Sample labeling and physicochemical characteristic of cobalt ferrite particles differing in metal 
concentration, ionic strength, and calcination temperatures are summarized in Table 2. The XRD diffraction patterns of the cobalt ferrite samples that correspond to crystallographic planes of (220), (311), (222), (400), (422), (511), and (440) are shown in Figures 5(a)-5(d). All the diffractograms are indexed regarding the standard JCPDS no. 01-077-0426 to the characteristic reflections of the cubic spinel phase. The relative intensity observed for the most prominent plane (311) increased as the ionic strength of the solution used for the preparation of cobalt ferrite particles decreased (Figure 5(b)) or as the annealing temperature increased up to $800^{\circ} \mathrm{C}$ (Figure $5(\mathrm{c})$ ). The crystallinity of the cobalt ferrite particles calcined at $400^{\circ} \mathrm{C}$ increased to $35 \%$ compared to the sample without annealing. Further increase in calcination temperature resulted in a decrease in the amorphous phase content from $65 \%$ to $47 \%$, as shown in Table 2 . Under identical annealing conditions, the crystallite size slightly increased with increasing ionic strength and metal concentration. The smallest crystallite size was observed for sample S4 with $I=1.25 \mathrm{M}$ annealed at $400^{\circ} \mathrm{C}$, as shown in Table 2 and Figure 5(b). For sample S6 obtained at ionic strength $I=5 \mathrm{M}$, the larger crystallite size was observed than for sample S4 with $I=1.25 \mathrm{M}$. The determined crystallite size is consistent with the particle size measured by DLS. The average hydrodynamic size of $\mathrm{CoFe}_{2} \mathrm{O}_{4}$ increased with increasing metal concentration and ionic strength from $46 \mathrm{~nm}$ for sample S4 to $50 \mathrm{~nm}$ for sample S6. It probably results from simultaneous nucleation of new particles at lower concentration and ionic strength, preventing further crystal growth. The higher ionic strength of the solution causes a reduction of zeta potential at a constant $\mathrm{pH}$, resulting in lower electrostatic stability and an increase of the hydrodynamic diameter of the particles. At the highest values of ionic strength and concentration, further crystallite growth was observed, while the hydrodynamic diameter of ferrite particles decreased, as a result of compensation of the double electric layer. At constant metal concentration and ionic strength, the particle size increased with increasing calcination temperature. The growth of $\mathrm{CoFe}_{2} \mathrm{O}_{4}$ particles with an increase in calcination temperature was confirmed by the crystallite size obtained by the Scherrer formula (Table 2).

For samples annealed at $400^{\circ} \mathrm{C}$, an additional peak at $2 \theta$ $31.7^{\circ}$ appeared due to the presence of a trace amount of $\alpha$ $\mathrm{Fe}_{2} \mathrm{O}_{3}$ phase. The impurity phase formation is not consistent with substituent concentration. Therefore, it can be mainly attributed to the synthesis or postsynthesis conditions of the samples. The presence of $\alpha-\mathrm{Fe}_{2} \mathrm{O}_{3}$ was previously reported in the literature for samples obtained by the chemical coprecipitation method [20]. Moreover, the trace presence of hematite was not observed for samples calcined at $800^{\circ} \mathrm{C}$. The lattice parameter was calculated for the (311) plane of the samples and was estimated as $8.37 \AA$ for the samples $\mathrm{S} 4-\mathrm{S} 9$ calcined at $400^{\circ} \mathrm{C}$ and $600^{\circ} \mathrm{C}$. The calculated lattice constant is comparable to the reported values $[21,22]$. The distribution of cations in the tetrahedral and octahedral sites depends on the thermal treatment and the synthesis conditions. A further decrease of the lattice constant to $8.35 \AA$ was observed for the samples S10-S12, which may suggest that $\mathrm{Fe}^{3+}$ ions in tetrahedral sites move to octahedral sites while $\mathrm{Co}^{2+}$ ions at octahedral sites move to tetrahedral sites during cation migration upon annealing at $800^{\circ} \mathrm{C}[23,24]$.

The influence of the synthesis parameters, e.g., calcination temperature, metal concentration, and ionic strength on the specific surface area and the pore volume of the obtained nanocrystallites, was also investigated. It can be noticed that the calcination temperature and the concentration of the metals during synthesis affected the BET specific surface area of the spinel ferrites. The surface area increased with a decrease in metal concentration and calcination temperature. The highest BET surface area of $23 \mathrm{~m}^{2} \cdot \mathrm{g}^{-1}$ revealed that sample $\mathrm{S} 4$ calcined at $400^{\circ} \mathrm{C}$, which also exhibited the largest pore volume of $0.012 \mathrm{~cm}^{3} \cdot \mathrm{g}^{-1}$ and the smallest crystallite size of $15 \mathrm{~nm}$.

Diffuse reflectance spectra for $\mathrm{CoFe}_{2} \mathrm{O}_{4}$ particles were transformed into the Kubelka-Munk function and are presented in Figure 6. All samples absorb in the range from $300 \mathrm{~nm}$ to $1000 \mathrm{~nm}$. At constant annealing temperature, an increase in metal concentration and ionic strength resulted in the preparation of particles with a more substantial absorbance within the UV-Vis range. The optical band gap value estimated from Tauc plots was found to slightly decrease from 1.80 for sample S4 obtained at $400^{\circ} \mathrm{C}$ with $I=1.25 \mathrm{M}$ to $1.60 \mathrm{eV}$ for sample S12 with higher metal concentration and calcined at $800^{\circ} \mathrm{C}$, as presented in Table 2 .

The Fourier transform infrared (FTIR) spectra for the obtained samples showed the characteristic features of spinel ferrite particles (see Figure 7). The absorption band observed in the range from $542 \mathrm{~cm}^{-1}$ to $551 \mathrm{~cm}^{-1}$ is assigned to the stretching vibrations of the tetrahedral metal-oxygen bond [23]. The peak at $875 \mathrm{~cm}^{-1}$ corresponds to the deformations of $\mathrm{Fe}-\mathrm{OH}$ groups and is manifested for all the obtained samples. The sharp peak at $1124 \mathrm{~cm}^{-1}$, which appeared for all cobalt ferrite particles calcined at $400-800^{\circ} \mathrm{C}$ and synthesized from metal sulphate precursors, corresponds to the symmetric stretching mode of sulphate anion $\left(1130-1080 \mathrm{~cm}^{-1}\right)$ chemisorbed by the metal's surface during the preparation procedure [25].

The absorption peak at $1435 \mathrm{~cm}^{-1}$ corresponds to bending vibrations of the $\mathrm{O}-\mathrm{H}$ bond. The appearance of bands around $2100-2370 \mathrm{~cm}^{-1}$ is due to the atmospheric $\mathrm{CO}_{2}$, which is adsorbed on the surface of NPs during the FTIR measurements [25]. The broad branch at $3450-3200 \mathrm{~cm}^{-1}$ corresponds to the $\mathrm{O}-\mathrm{H}$ stretching vibrations ascribed to water [26].

Figure 8 shows the hysteresis loops obtained from VSM measurements for the prepared $\mathrm{CoFe}_{2} \mathrm{O}_{4}$ particles differing in ionic strength, concentration of metals, and calcination temperature during the ferrite synthesis. The saturation magnetization $\left(M_{\mathrm{s}}\right)$, remanent magnetization $\left(M_{\mathrm{r}}\right)$, and coercivity $\left(H_{c}\right)$ of cobalt ferrite nanoparticles calcined at $400^{\circ} \mathrm{C}$, $600^{\circ} \mathrm{C}$, and $800^{\circ} \mathrm{C}$ are presented in Table 2. The observed magnetic properties depend on pretreatment conditions. With an increase of calcination temperature to $800^{\circ} \mathrm{C}$, the saturation magnetization for the samples with $I=1.25 \mathrm{M}$ decreased from $51 \mathrm{emu} \cdot \mathrm{g}^{-1}$ to $45 \mathrm{emu} \cdot \mathrm{g}^{-1}$ (see samples S4, S7, and S10). For samples obtained in solutions with higher ionic strength $I=2.5 \mathrm{M}$ and $I=5 \mathrm{M}$, the effect of calcination 


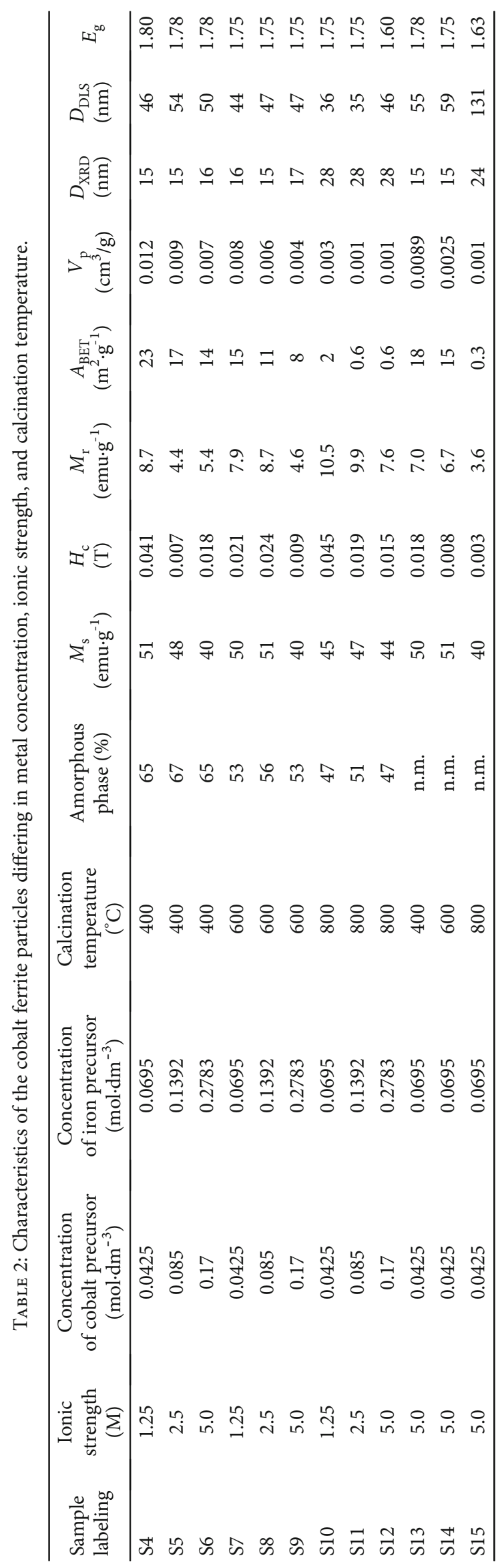



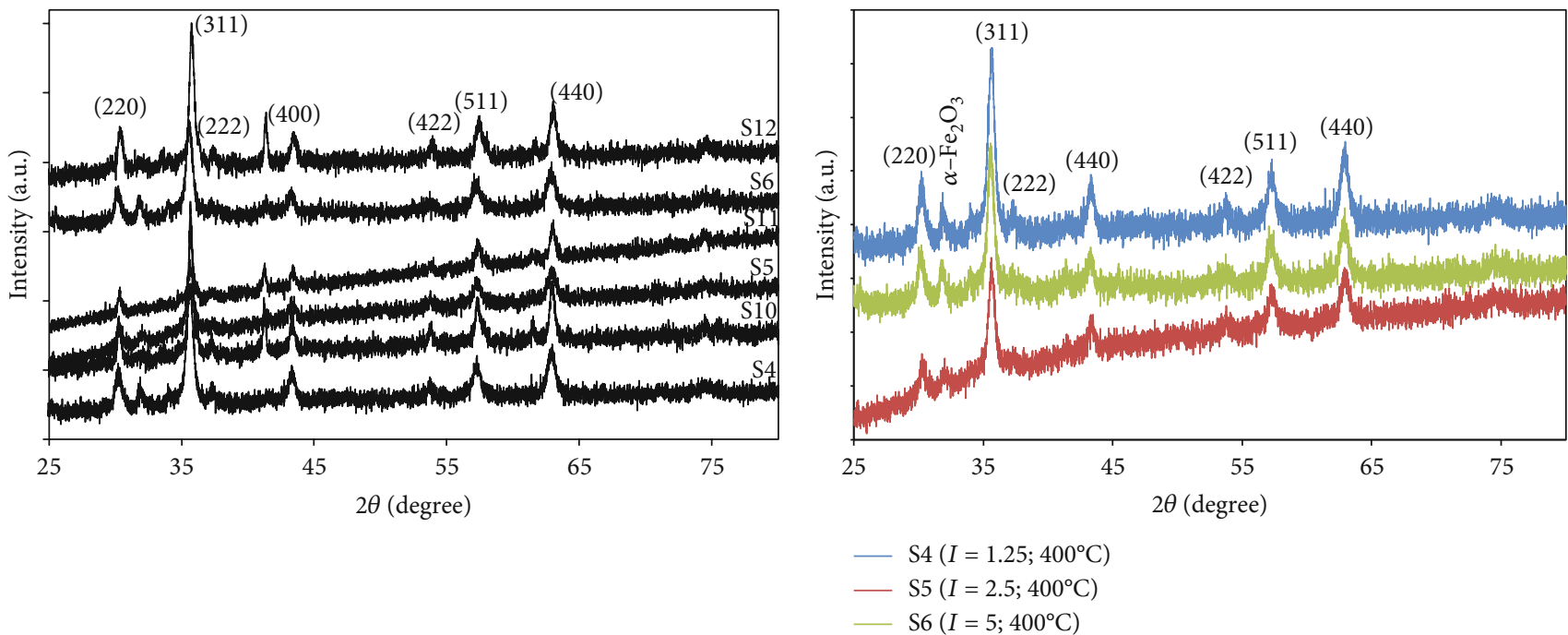

(a)

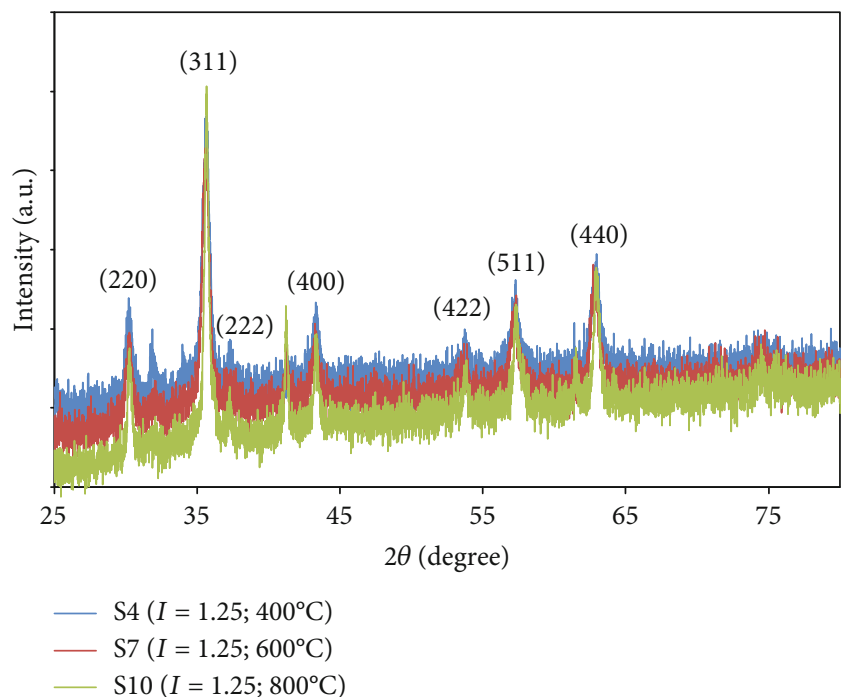

(b)

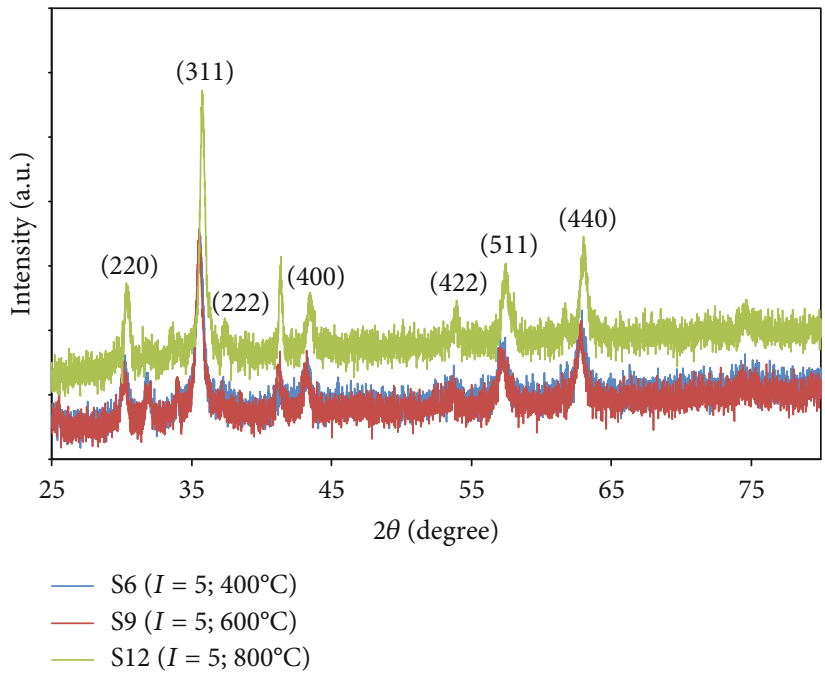

(c)

(d)

FIgure 5: X-ray diffraction patterns of the as-prepared $\mathrm{CoFe}_{2} \mathrm{O}_{4}$ particles (a), calcined at $400^{\circ} \mathrm{C}$ with different ionic strength (b), with ionic strength $I=1.25 \mathrm{M}$ and different annealing temperatures (c), and with ionic strength $I=5 \mathrm{M}$ and different annealing temperatures (d).

temperature on saturation magnetization was not unambiguously confirmed despite the observed increase in crystallite size with increased annealing temperature. The ionic strength and concentration of metals in the solution during the synthesis of $\mathrm{CoFe}_{2} \mathrm{O}_{4}$ particles have a significant influence on $M_{\mathrm{s}}$ and coercivity values.

The highest saturation magnetization revealed samples S4, S7, and S8 with ionic strength $I=1.25 \mathrm{M}$ and $I=2.5 \mathrm{M}$ calcined at $400^{\circ} \mathrm{C}$ and $600^{\circ} \mathrm{C}$, respectively. For samples calcined in $400^{\circ} \mathrm{C}$ and $600^{\circ} \mathrm{C}$, an increase of ionic strength resulted in a $20 \%$ lower $M_{s}$ value. The resulting values of $M_{\mathrm{s}}$ for $800^{\circ} \mathrm{C}$ are not significantly different between samples with different $I$. The coercivity and remanent magnetization also decreased for samples obtained in solutions with the highest ionic strength, as shown in Table 2. The values of $H_{\mathrm{c}}$ and $M_{\mathrm{r}}$ were significantly different between samples calcined in the same temperature for all three series, in contrast to the values of $M_{\mathrm{s}}$ for $T_{\text {calc }}=800^{\circ} \mathrm{C}$. In the group of samples calcined in the same temperatures, $H_{\mathrm{c}}$ and $M_{\mathrm{r}}$ values were always correlated and were the lowest for the samples with the highest ionic strength and concentration of metal precursors.

To confirm the effect of concentration and ionic strength of metal salt solution, additional samples S13-S15 with different ionic strengths controlled by adding the appropriate quantity of $\mathrm{NaClO}_{5}$ with the same concentration of metal precursor salts as for sample S4 were obtained. The ionic strength during precipitation of metals had a significant impact on coercivity, whereas the concentration of metals influenced saturation magnetization and morphology of the obtained cobalt ferrite particles. For samples S13-S14 calcined at $400^{\circ} \mathrm{C}$ and $600^{\circ} \mathrm{C}$, high ionic strength of the solution 


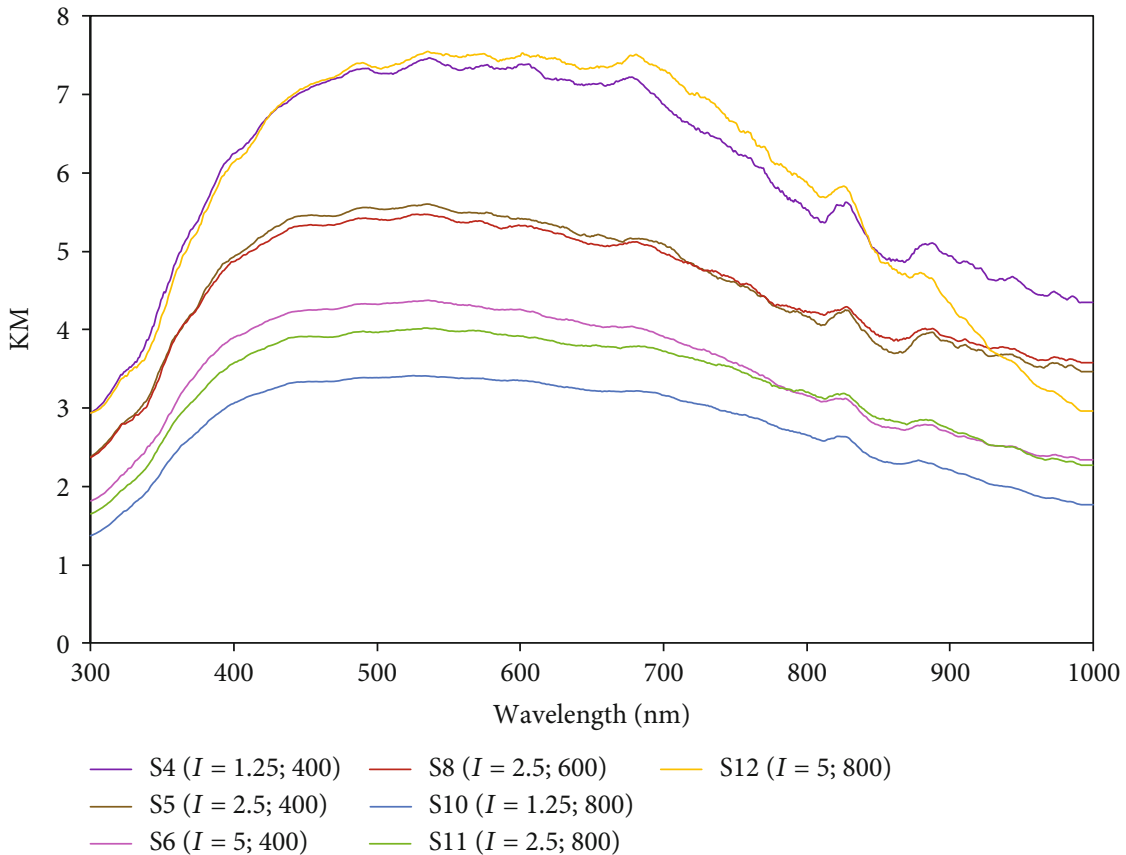

Figure 6: DR/UV-Vis spectra presented as the Kubelka-Munk function for $\mathrm{CoFe}_{2} \mathrm{O}_{4}$ particles calcined at $400-800^{\circ} \mathrm{C}$ with ionic strength $I=1.25 \div 5.0 \mathrm{M}$.

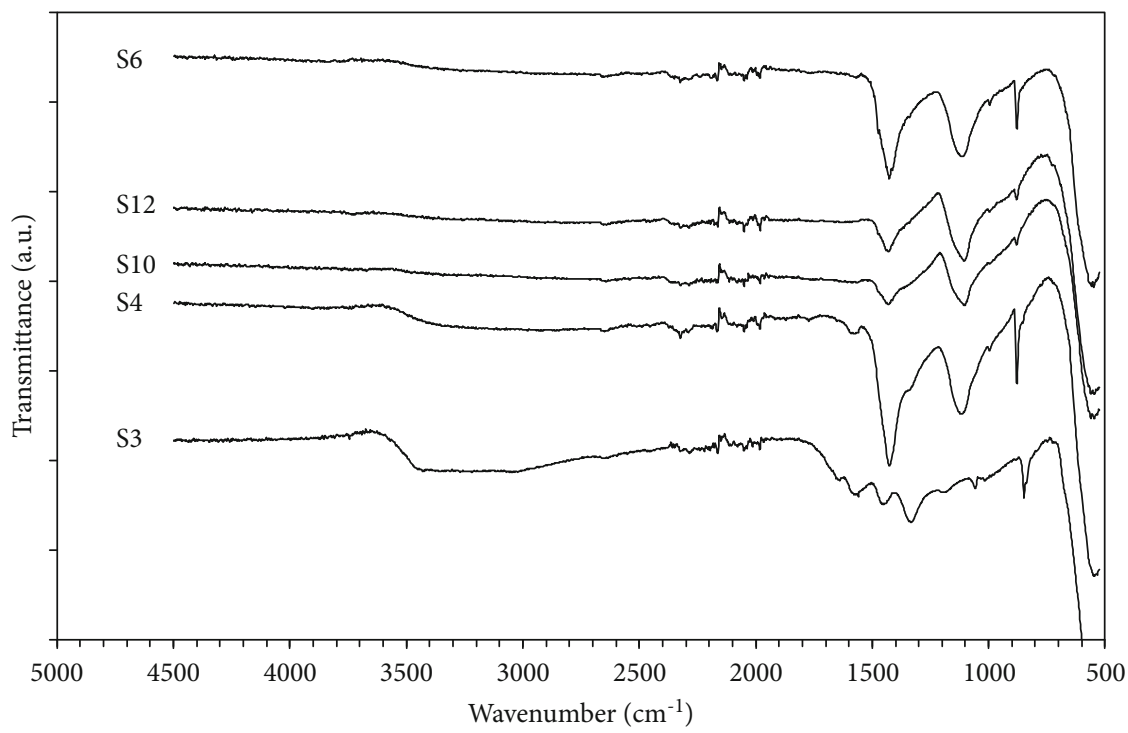

FIgURE 7: Fourier transform infrared (FTIR) spectra for the $\mathrm{CoFe}_{2} \mathrm{O}_{4}$ particles.

and lower concentration of metals limited the particle growth by Ostwald ripening. The average crystallite size and the average hydrodynamic size of $\mathrm{CoFe}_{2} \mathrm{O}_{4}$ particles were about $15 \mathrm{~nm}$ and $50 \mathrm{~nm}$, respectively.

In this regard, changing the concentration of metals may be regarded as a simple method to optimize the morphology of cobalt ferrite particles. At the same ionic strength $I=5 \mathrm{M}$, lower concentration of metal salts resulted in a higher BET surface area and smaller crystallite size of cobalt ferrites, as shown in Table 2 for samples S13 and S6 as well as S14 and S9. Moreover, the concentration of metals affected the satura- tion magnetization value, whereas the ionic strength determined the coercivity of obtained $\mathrm{CoFe}_{2} \mathrm{O}_{4}$ particles. At the same ionic strength and lower concentration, the $M_{\mathrm{s}}$ value was markedly higher for S13 and S14 compared to S6 and S9 samples. The coercivity and remanent magnetization increased for samples S4 and S7 with lower ionic strength $I=1.25 \mathrm{M}$ compared to $\mathrm{S} 13$ and $\mathrm{S} 14$ samples with $I=5 \mathrm{M}$. The highest annealing temperature of $800^{\circ} \mathrm{C}$ also greatly affected the morphology of $\mathrm{CoFe}_{2} \mathrm{O}_{4}$ particles and, as a result, changes in magnetic properties. Therefore, the resulting values of $M_{\mathrm{s}}$ and $M_{\mathrm{r}}$ are not significantly different between 


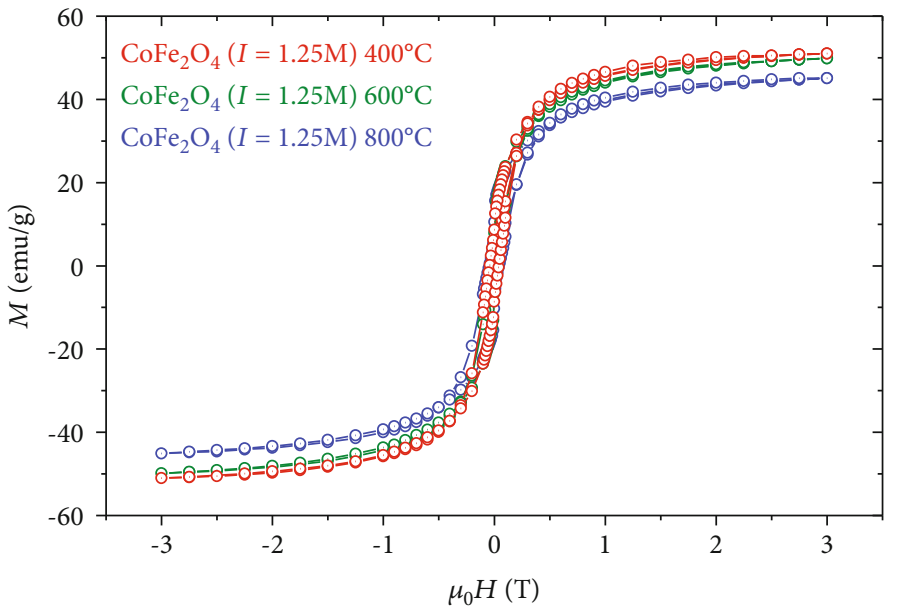

(a)

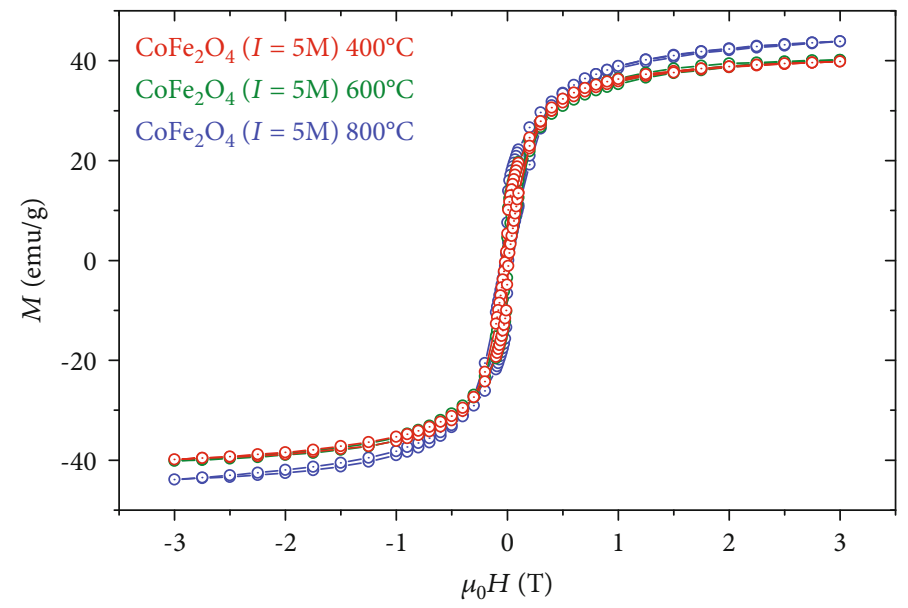

(b)

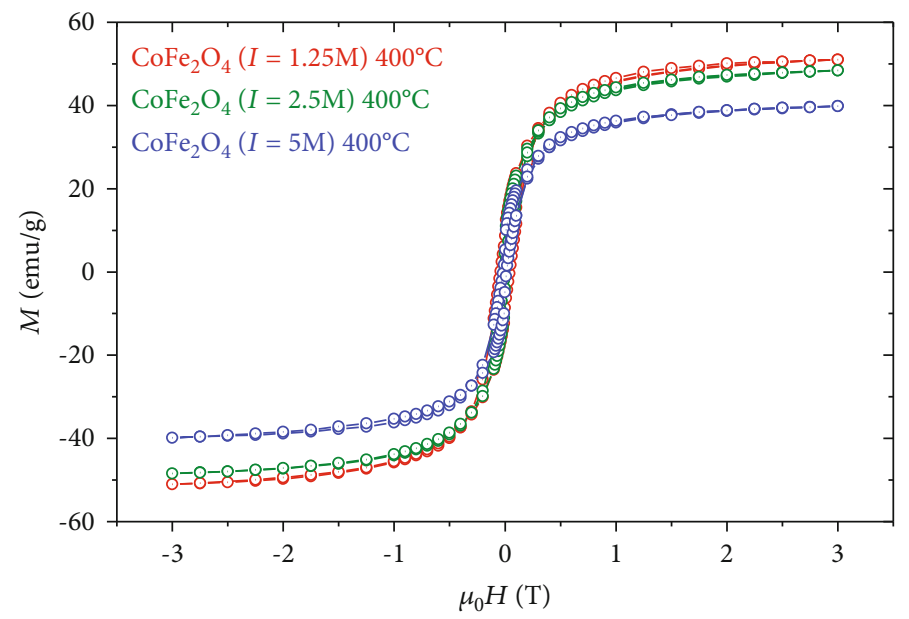

(c)

FIgURE 8: Continued. 


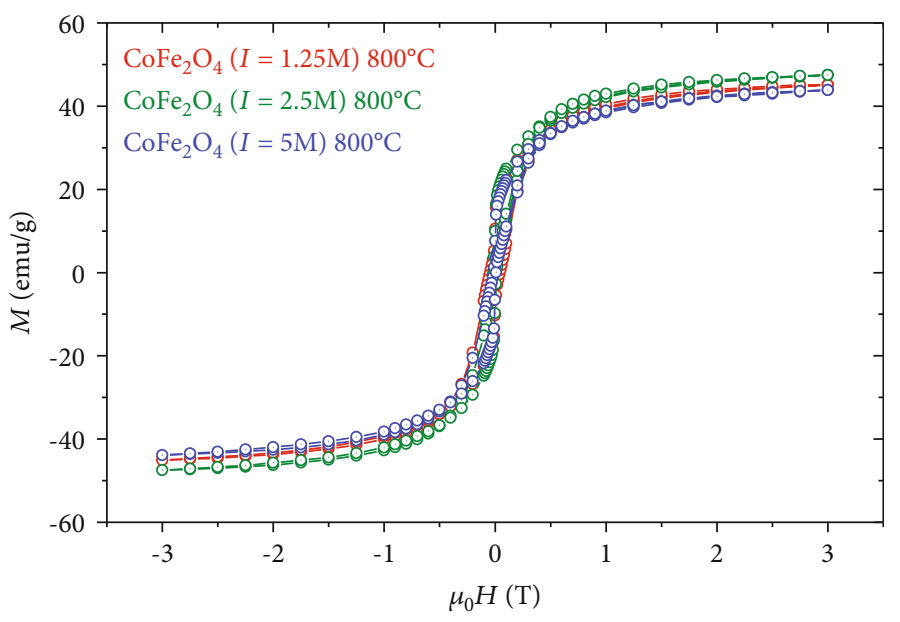

(d)

Figure 8: Magnetic hysteresis loops of $\mathrm{CoFe}_{2} \mathrm{O}_{4}$ calcined at $400-800^{\circ} \mathrm{C}$ with ionic strength $I=1.25 \mathrm{M}$ (a), calcined at $400-800^{\circ} \mathrm{C}$ with ionic strength $I=5 \mathrm{M}(\mathrm{b})$, calcined at $400^{\circ} \mathrm{C}$ with different ionic strength (c), and calcined at $800^{\circ} \mathrm{C}$ with different ionic strength $I=1.25 \div 5 \mathrm{M}(\mathrm{d})$.

the samples calcined in $800^{\circ} \mathrm{C}$ due to similar particle size, specific surface area, and lattice constant parameters. Meanwhile, the $H_{c}$ value depended on the ionic strength of the precursor's solution.

\section{Conclusions}

The obtained results indicate a significant influence of a precursor type, its concentration, and ionic strength of the solution on the morphology and magnetic properties of cobalt ferrite particles prepared by the hydrothermal method. It was found that the chemistry of metal precursors is correlated with magnetic properties. The $\mathrm{CoFe}_{2} \mathrm{O}_{4}$ particles from metal sulphate precursors showed the highest saturation magnetization, the lowest remanent magnetization, and the lowest coercive values.

The ionic strength of the metal solution controls the coercivity, whereas the concentration of metals strongly affects the saturation magnetization and morphology of the obtained metal ferrite particles. At the same ionic strength $I=5 \mathrm{M}$, the lower concentration of metal salts resulted in a higher BET surface area and smaller crystallite size of cobalt ferrite particles. The concentration of metals and, as a result, the morphology of the cobalt ferrite NPs influenced the saturation magnetization, which was enhanced for the samples ( $I=$ const, $T=$ const) obtained from diluted metal precursor solution. The ionic strength determined the coercivity of the $\mathrm{CoFe}_{2} \mathrm{O}_{4}$ particles, which increased for the samples with the lowest ionic strength $I=1.25 \mathrm{M}$. The highest annealing temperature of $800^{\circ} \mathrm{C}$ greatly affected the morphology of $\mathrm{CoFe}_{2} \mathrm{O}_{4}$ particles and, as a result, changes in magnetic properties. The increase of calcination temperature resulted in a larger particle size and decreased BET specific surface area of the spinel ferrites. Despite higher crystallinity of the $\mathrm{CoFe}_{2} \mathrm{O}_{4}$ particles annealed at $800^{\circ} \mathrm{C}$, the saturation magnetization value decreased. Furthermore, it can be affected by the distribution of cations between the interstitial sites.
The particle size of cobalt ferrite can be adjusted and stabilized against ripening by control of ionic strength, annealing temperature, and concentration of metal salts in a precipitation medium. The $\mathrm{CoFe}_{2} \mathrm{O}_{4}$ sample obtained from diluted metal precursor solution with the lowest ionic strength and calcined in temperatures of $400^{\circ} \mathrm{C}$ $600^{\circ} \mathrm{C}$ possessed a small particle size of $15 \mathrm{~nm}$ and higher specific surface area and revealed the highest magnetic properties $\left(M_{\mathrm{s}}\right.$ and $H_{\mathrm{c}}$ values). In this regard, changing the metal concentration, ionic strength, and annealing temperature may be regarded as a simple method to optimize the morphology and, as a result, magnetic properties of the ferrite particles, which may otherwise be difficult.

\section{Data Availability}

All the results and data used to support the findings of this study are included within the article.

\section{Conflicts of Interest}

The authors declare that they have no conflict of interest.

\section{Authors' Contributions}

The contributions of the authors involved in this study are as follows: conceptualization, Anna Zielińska-Jurek; synthesis, Izabela Malinowska; formal analysis, Izabela Malinowska, Zuzanna Sobczak, and Eryka Mrotek; funding acquisition, Anna Zielińska-Jurek; investigation, Izabela Malinowska; methodology, Anna Zielińska-Jurek and Tomasz Klimczuk; project administration, Anna ZielińskaJurek; writing-original draft, Anna Zielińska-Jurek; and writing-review and editing, Anna Zielińska-Jurek and Tomasz Klimczuk. 


\section{Acknowledgments}

This research was financially supported by the Polish National Science Centre (Grant No. NCN2016/23/D/ST5/01021).

\section{References}

[1] D. S. Mathew and R. S. Juang, "An overview of the structure and magnetism of spinel ferrite nanoparticles and their synthesis in microemulsions," Chemical Engineering Journal, vol. 129, no. 1-3, pp. 51-65, 2007.

[2] C. Murugesan, L. Okrasa, and G. Chandrasekaran, "Structural AC conductivity impedance and dielectric study of nanocrystalline $\mathrm{MFe}_{2} \mathrm{O}_{4}(\mathrm{M}=\mathrm{Mg}$, Co or $\mathrm{Cu})$ spinel ferrites," Journal of Materials Science: Materials in Electronics, vol. 28, no. 17, pp. 13168-13175, 2017.

[3] R. Valenzuela, "Novel applications of ferrites," Physics Research International, vol. 2012, Article ID 591839, 9 pages, 2012.

[4] V. S. Kumbhar, A. D. Jagadale, N. M. Shinde, and C. D. Lokhande, "Chemical synthesis of spinel cobalt ferrite $\left(\mathrm{CoFe}_{2} \mathrm{O}_{4}\right)$ nano-flakes for supercapacitor application," Applied Surface Science, vol. 259, pp. 39-43, 2012.

[5] N. Sivakumar, A. Narayanasamy, B. Jeyadevan, R. J. Joseyphus, and C. Venkateswaran, "Dielectric relaxation behaviour of nanostructured Mn - Zn ferrite," Journal of Physics D: Applied Physics, vol. 41, no. 24, p. 245001, 2008.

[6] A. J. Rondinone, C. Liu, and Z. J. Zhang, "Determination of magnetic anisotropy distribution and anisotropy constant of manganese spinel ferrite nanoparticles," Journal of Physical Chemistry B, vol. 105, no. 33, pp. 7967-7971, 2001.

[7] M. Sugimoto, “The past, present, and future of ferrites," Journal of the American Ceramic Society, vol. 82, no. 2, pp. 269280, 1999.

[8] A. Zielinska-Jurek, Z. Bielan, S. Dudziak et al., "Design and application of magnetic photocatalysts for water treatment. The effect of particle charge on surface functionality," Catalysts, vol. 7, no. 12, p. 360, 2017.

[9] L. Néel, "Antiferromagnetism and ferrimagnetism," Proceedings of the Physical Society Section A, vol. 65, no. 11, pp. 869885, 1952.

[10] L. Ai and J. Jiang, "Influence of annealing temperature on the formation, microstructure and magnetic properties of spinel nanocrystalline cobalt ferrites," Current Applied Physics, vol. 10, no. 1, pp. 284-288, 2010.

[11] M. Houshiar, F. Zebhi, Z. J. Razi, A. Alidoust, and Z. Askari, "Synthesis of cobalt ferrite (CoFe2O4) nanoparticles using combustion, coprecipitation, and precipitation methods: A comparison study of size, structural, and magnetic properties," Journal of Magnetism and Magnetic Materials, vol. 371, pp. 43-48, 2014.

[12] A. Pradeep, P. Priyadharsini, and G. Chandrasekaran, "Sol-gel route of synthesis of nanoparticles of $\mathrm{MgFe} 2 \mathrm{O} 4$ and XRD, FTIR and VSM study," Journal of Magnetism and Magnetic Materials, vol. 320, no. 21, pp. 2774-2779, 2008.

[13] L. Wang, J. Li, Y. Wang, L. Zhao, and Q. Jiang, “Adsorption capability for Congo red on nanocrystalline MFe2O4 $(\mathrm{M}=$ $\mathrm{Mn}, \mathrm{Fe}, \mathrm{Co}, \mathrm{Ni}$ ) spinel ferrites," Chemical Engineering Journal, vol. 181-182, pp. 72-79, 2012.

[14] V. Pillai and D. O. Shah, "Synthesis of high-coercivity cobalt ferrite particles using water-in-oil microemulsions," Journal of Magnetism and Magnetic Materials, vol. 163, no. 1-2, pp. 243-248, 1996.

[15] J. Wagner, T. Autenrieth, and R. Hempelmann, "Core shell particles consisting of cobalt ferrite and silica as model ferrofluids [CoFe2O4-SiO2 core shell particles]," Journal of Magnetism and Magnetic Materials, vol. 252, pp. 4-6, 2002.

[16] S. Ammar, A. Helfen, N. Jouini et al., "Magnetic properties of ultrafine cobalt ferrite particles synthesized by hydrolysis in a polyol medium," Journal of Materials Chemistry, vol. 11, no. 1, pp. 186-192, 2001.

[17] R. Ianoş, M. Bosca, and R. Lazău, "Fine tuning of $\mathrm{CoFe}_{2} \mathrm{O}_{4}$ properties prepared by solution combustion synthesis," Ceramics International, vol. 40, no. 7, pp. 10223-10229, 2014.

[18] Y. Zhao, Y. Xu, J. Zeng et al., "Low-crystalline mesoporous $\mathrm{CoFe}_{2} \mathrm{O}_{4} / \mathrm{C}$ composite with oxygen vacancies for high energy density asymmetric supercapacitors," RSC Advances, vol. 7, no. 87, pp. 55513-55522, 2017.

[19] B. J. Rani, M. Ravina, B. Saravanakumar et al., "Ferrimagnetism in cobalt ferrite $\left(\mathrm{CoFe}_{2} \mathrm{O}_{4}\right)$ nanoparticles," Nano-Structures \& Nano-Objects, vol. 14, pp. 84-91, 2018.

[20] M. G. Naseri, E. B. Saion, H. A. Ahangar, A. H. Shaari, and M. Hashim, "Simple synthesis and characterization of cobalt ferrite nanoparticles by a thermal treatment method," Journal of Nanomaterials, vol. 2010, Article ID 907686, 8 pages, 2010.

[21] R. Ianoș, "Highly sinterable cobalt ferrite particles prepared by a modified solution combustion synthesis," Materials Letters, vol. 135, pp. 24-26, 2014.

[22] B. Babić-Stojić, V. Jokanović, D. Milivojević et al., "Magnetic and structural studies of $\mathrm{CoFe}_{2} \mathrm{O}_{4}$ nanoparticles suspended in an organic liquid," Journal of Nanomaterials, vol. 2013, Article ID 741036, 9 pages, 2013.

[23] L. Kumar, P. Kumar, A. Narayan, and M. Kar, "Rietveld analysis of XRD patterns of different sizes of nanocrystalline cobalt ferrite," International Nano Letters, vol. 3, no. 1, 2013.

[24] V. Bartůněk, D. Sedmidubský, Š. Huber, M. Švecová, P. Ulbrich, and O. Jankovský, "Synthesis and properties of nanosized stoichiometric cobalt ferrite spinel," Materials, vol. 11, no. 7, p. 1241, 2018.

[25] G. Lefevre, "In situ Fourier-transform infrared spectroscopy studies of inorganic ions adsorption on metal oxides and hydroxides," Advances in Colloid and Interface Science, vol. 107, no. 2-3, pp. 109-123, 2004.

[26] L. Frolova, A. Derimova, and T. Butyrina, "Structural and magnetic properties of cobalt ferrite nanopowders synthesis using contact non-equilibrium plasma," Acta Physica Polonica $A$, vol. 133, no. 4, pp. 1021-1023, 2018. 